\title{
ITGB2 as a Novel Biomarker Correlating With Prognosis and Immune Infiltrates in Ovarian Cancer
}

\section{chanyuan li ( 0 495482613@qq.com )}

Department of Gynecology and Obstetrics, The Fifth Affiliated Hospital of Sun Yat-Sen University, Zhuhai 519000,Guangdong, China 2 Department of Gynecologic Oncology, State Key Laboratory of Oncology in South China, Collaborative Innovation Center for Ca https://orcid.org/0000-0003-1485-670X

\section{Ting Wan}

Department of Gynecologic Oncology, State Key Laboratory of Oncology in South China, Collaborative Innovation Center for Cancer Medicine, Sun Yat-Sen University Cancer Center, Guangzhou 510060, Guangdong, China

\section{Ting Deng}

Department of Gynecologic Oncology, State Key Laboratory of Oncology in South China, Collaborative Innovation Center for Cancer Medicine, Sun Yat-Sen University Cancer Center, Guangzhou 510060, Guangdong, China

\section{Junya Cao}

Department of Gynecologic Oncology, State Key Laboratory of Oncology in South China, Collaborative Innovation Center for Cancer Medicine, Sun Yat-Sen University Cancer Center, Guangzhou 510060, Guangdong, China

\section{He Huang}

Department of Gynecologic Oncology, State Key Laboratory of Oncology in South China, Collaborative Innovation Center for Cancer Medicine, Sun Yat-Sen University Cancer Center, Guangzhou 510060, Guangdong, China

Jihong Liu

1 Department of Gynecology and Obstetrics, The Fifth Affiliated Hospital of Sun Yat-Sen University, Zhuhai 519000,Guangdong, China 2 Department of Gynecologic Oncology, State Key Laboratory of Oncology in South China, Collaborative Innovation Center for $\mathrm{Ca}$

\section{Research}

Keywords: ovarian cancer, prognosis, immune infiltration, TAMs, ITGB2

Posted Date: March 1st, 2021

DOI: https://doi.org/10.21203/rs.3.rs-263949/v1 
License: (c) (i) This work is licensed under a Creative Commons Attribution 4.0 International License. Read Full License 


\section{Abstract}

Background: Epithelial ovarian cancer is nowadays one of the malignancies in women, this study aimed to identify novel biomarkers to predict prognosis and immunotherapy efficacy.

Methods: The differentially expressed genes (DEGs) obtained from online database Gene Expression Omnibus (GEO)were screened via GEO2R and Venn diagram software, gene enrichment was analysed by Gene Ontology(GO) function and Kyoto Encyclopedia of Genes and Genomes(KEGG), then protein protein interaction(PPI)network and Cytoscape software were used to confirm the genes closely related to ovarian cancer. Survival analysis of hub genes were obtained from Kaplan-Meier plotter, with their differential expression in specimen validated by Gene Expression Profiling Interactive Analysis (GEPIA) and an integrated repository portal for tumor-immune system interactions (TISIDB). Finally, we used the Tumor Immune Estimation Resource 2.0 (TIMER2.0) and application Estimate the Proportion of Immune and Cancer cells (EPIC) to search the immune infiltration characteristics of the genes.

Results: 355 DEGs between epithelial ovarian cancer and normal ovarian tissue were screened out. These DEGs were associated with extracellular exosome, bicellular tight junction and cell-cell junction, and remarkably enriched in molecules of cell adhesion and leukocyte transendothelial migration activity. Ten hub genes were identified via protein protein interaction (PPI) network: PTAFR, HLA-DRA, OAS2, OAS3, PTPN6, LYN, VAMP8, IRF6, ITGB2, CD47. Furthermore, the Kaplan-Meier plotter was conducted, overexpression of four genes was positively connected to poor prognosis in ovarian cancer:OAS2, OAS3, ITGB2, CD47, which were also correlated with immune infiltrates in ovarian cancer and had the highest degree of correlation with tumor associated macrophages (TAMs) infiltration, among which ITGB2 was highly correlated with TAMs infiltration level.

Conclusion: ITGB2, OAS2, OAS3, and CD47 are upregulated with unfavorable prognosis in ovarian cancer, and ITGB2 may act as a novel prognostic biomarker with immune infiltration values.

\section{Introduction}

Epithelial ovarian cancer is nowadays one of the malignancies in women with a high degree of malignancy, recurrence rate and poor prognosis. Current treatment for II-IV of epithelial ovarian cancer consist of initial complete cytoreductive surgery supplemented with platinum-based chemotherapy, however, the side effects and drug resistance of chemotherapy affect the prognosis of ovarian cancer. Despite the substantial progress in targeted therapy, the treatment of ovarian cancer (OV) has changed to combination treatments such as targeted therapy, gene therapy and immunotherapy, which will become the trend of ovarian cancer treatment. ${ }^{1}$

Based on the immunoscore and contexture of tumors, tumors can be divided into four types: hot, cold immunosuppressed and excluded tumors. ${ }^{2}$ Ovarian cancer is a type of cold tumor, of which T lymphocyte infiltration is rare, and the infiltrating $T$ lymphocytes cannot recognize all tumor antigens, so the treatment of immune checkpoint such as PD-1 and CTLA-4 inhibitors has little benefit . ${ }^{3,4}$ In fact, the tumor micro- 
environment not only includes the tumor itself and the matrix, but tumor-related macrophages, suppressor cells of myeloid origin and tumor-related fibrosis, while the genetic mutation load of these immune cells affects the immune characteristics and is related to the tumor's response to immunotherapy and prognosis. ${ }^{5}$

Our study aimed to investigate the influence of abnormal immune genome expression on the prognosis of ovarian cancer and its potential effect. Exploration of potential immune-related genes and their impact on the tumor microenvironment, which will provide ideas to new immuno -therapy targets and immune characteristics of patients with ovarian cancer, we initially downloaded GSE36668 and GSE66957 from the online database GEO via GEO2R and Venn diagram to screen out DEGs, next performed gene enrichment analysis of DEGs by the Database for Annotation, Visualization and Integrated Discovery (DAVID) database, then we used PPI network and Cytoscape software to confirm the genes related to ovarian cancer, and predicted the prognosis through Kaplan-Meier plotter, validating the hub genes with GEPIA, subsequently, ITGB2, OAS2, OAS3, and CD47 were identified remarkably connected with the unfavorable prognosis of epithelial ovarian cancer, Finally, the TIMER2.0 and EPIC were applied to explore the immune infiltration characteristics of genes.

\section{Materials And Methods}

\section{Microarray Data}

We obtained gene expression datasets from GEO online database (https://www.ncbi.nlm.nih. gov/geo/). GSE36668 and GSE66957 expression profiles were chose.

\section{DEGs identification}

The DEGs between serous ovarian cancer and normal ovarian samples were identified through GEO2R online tools (https://www.ncbi.nlm. nih.gov/geo/geo2r/). Genes satisfied the cutoff criteria, adjusted $\mathrm{P}<$ 0.05 and $|\log F C| \geq 2.0$, were selected as DEGs. We defined $\log F C>2$ as upregulated genes, while the $\log F C<$ -2 to be down-regulated genes, and the Venn diagram was conducted to identify the intersecting part of DEGs. (bioinformatics.psb.ugent.be/webtools/Venn/).

\section{GO and KEGG pathway analysis}

DAVID (https://david.ncifcrf.gov/), a tool used to identify the biological function of DEGs and visualize their enrichment, then we used GO analysis to define genes and their products, and identified unique biological functions. KEGG is a collection of databases who can process genome's complex information, chemicals as well as biological pathways. $P<0.01$ and counts of genes $\geq 10$ were viewed as statistically significant.

\section{Construction of PPI network and identification of hub genes}


To analyze and identify the possible PPI relationship, DEGs identified before were schemed via database STRING (Search Tool for the Retrieval of Interacting Genes) (http://string-db.org/). Pairs of PPI were accumulated with a combined score 0.4. Then the Cytoscape software (www.cytoscape.org/) , a online tool visualizing the PPI network was performed, while the degree of each protein node was calculated by CytoHubba so as to verify the hub genes.

\section{Prognosis analysis of hub genes}

The survival and clinical data of genes were calculated by Kaplan-Meier plotter (http://kmplot.com/analysis/) and TISIDB: an comprehensive portal for tumor immune system interaction (http://cis.hku.hk/TISIDB/index.php) . The expression and correlation between gene and infiltrating immune cells surface markers were verified by GEPIA (http://gepia.cancer-pku.cn/index.html) on the basis of adjusted hazard ratio (HR) with $95 \%$ confidence intervals and log-rank P-value.

\section{Immune infiltration analysis of genes}

In order to identify the prognosis related genes' correlation with the abundances of tumor-infiltrating immune cells, the online tool EPIC(https://gfellerlab.shinyapps.io/EPIC_1-1/)and TIMER2.0 (http://timer.cistrome.org) was used via the corresponding functional modules.

\section{Results}

\section{DEGs identification.}

Two expression profiles (GSE36668 and GSE66957) were selected, GSE36668 contained 4 serous ovarian cancer and 4 normal samples, while GSE66957 included 57 serous ovarian cancer and 12 normal ovarian specimens. On the basis of the standard of $\mathrm{P}<0.05$ and $|\log \mathrm{FC}| \geq 2.0,1428 \mathrm{DEG}$ were confirmed from GSE36668, with 749 upregulated and 679 downregulated genes. In profile GSE66957, 2,579 DEGs were identified; of which 1852 genes were upregulated and 727 genes were downregulated. All DEGs were screened by comparing epithelial ovarian cancer to normal ovarian tissues. Then Venn analysis was conducted to obtain the intersection part of the DEGs (Figure 1). In the end, 355 DEGs were significantly differentially expressed in two profiles, consisting of 314 upregulated genes and 41 downregulated genes.

\section{GO and KEGG pathway analyses of DEGs}

GO function and KEGG pathway enrichment of DEGs were conducted via the tool DAVID (Table 1). GO terms of DEGs were separated into CC and BP categories. The significantly upregulated terms enriched in cell component, including extracellular exosome, apical plasma membrane, bicellular tight junction, cell to cell junction, basolateral plasma membrane, lateral plasma membrane and extracellular matrix organization. Besides, the KEGG pathway revealed that DEGs were substantially enriched in molecules of cell adhesion, leukocyte transendothelial migration activity and staphylococcus aureus infection. In summary, these DEGs may play a role in cells' communication and transition. 


\section{Ten hub genes were identified via PPI}

DEGs' protein interactions were predicted through STRING. Total amount of closely connected 89 nodes includes 10 highest degree nodes contained in PPI network as listed in Figure 2. Top ten genes assessed by highest node degree in PPI network were confirmed (Table2), including PTAFR,HLA-DRA, OAS2, OAS3, PTPN6, LYN, VAMP8, IRF6, ITGB2, CD47. All of these genes were upregulated in ovarian cancer.

To identify the prognostic value of the genes above, the tool Kaplan-Meier plotter platform was performed. Higher expression of four hub genes: ITGB2, OAS2, OAS3, CD47 were connected with poor overall survival of ovarian cancer patients.(Figure3), while OAS2,OAS3 were related to ovarian cancer grade, which means that overexpression of OAS2 and OAS3 were associated with higher grade in ovarian cancer, and they may be important parameters that affect grade in OV patients(Figure4). Furthermore, we used GEPIA to verify that these genes were overexpressed in ovarian cancer compared to normal ovarian. (Figure5)

\section{ITGB2 was correlated with TAMs infiltration in ovarian cancer}

To analyse the classification of tumor-infiltrating immune cells in our samples, we first used TIMER and EPIC to figure up the immune cell types, the results showed the immune response in two groups has been suppressed to a certain extent,while macrophage particularly macrophageM2accounts a large proportion in these immune cells. (Figure6)Then we assessed the correlation between four genes and major infiltrating immune cells in ovarian cancer, such as CD8+ TcellखCD4+ Tcell,B cell and macrophages, we found that these genes were correlated with immune infiltrates in ovarian cancerand had the highest degree of correlation with macrophage infiltration, especially tumor- associated macrophages, among which ITGB2 is strongly correlated with macrophageM2 infiltration level( $r=0.814, p=2.58 \mathrm{e}-60)$ (Figure7-8). Otherwise, the relationships between genes expression and various markers of immune cells was verified via GEPIA.(Table3) These results indicated that ITGB2 may regulate the polarization of M2macrophage and its differentiation into TAMs, which contributes to ovarian carcinogenesis. In addition, ITGB2 also could be an important immune infiltration related gene in other human cancer.(Figure9凶

\section{Discussion}

Immunotherapy in ovarian cancer has not been effective as expected now, due to the immunosuppression and antitumor activity of the immune system, in order to stimulate the innate arm of immune system ,further studies on immunotherapy are supposed to focus on restoring the immune responsiveness of the tumor micro-environment and targeting heterogeneous tumors completely. ${ }^{6}$

The stromal cells in the metastatic microenvironment of ovarian cancer include fibroblasts, adipocytes, mesothelial cells and immune cells, among which macrophages occupy most. ${ }^{7,8}$ Tumor-associated macrophages(TAMs)show different phenotypic activation status between two categories depending on the cellular context: tumoricidal macrophages M1and regenerative macrophages M2, TAMs in ovarian cancer most closely resemble M2- polarized macrophages. ${ }^{9,10}$ The function of TAMs considered to act as 
an immunosuppressive role in tumor microenvironment, displaying the function promoting tumor invasion, growth and metastasis. ${ }^{11}$ Depending on the analysis of TIMER and EPIC, macrophage was found occupied a large proportion in the ovarian cancer matrix, and it is possibly that M2-polarized macrophage plays a vital role in the occurrence and unfavorable prognosis of ovarian cancer.

In our study, 355 DEGs between epithelial ovarian cancer and normal ovarian tissue were screened out from the GEO. DEGs were closely releated to the BP terms such as cell component, including extracellular exosome, apical plasma and basolateral membrane, bicellular tight junction, cell to cell junction, and significantly enriched in the KEGG terms as molecules of cell adhesion and leukocyte transendothelial migration activity. Ten hub genes were identified via the construction of a PPI network, including PTAFR,HLA-DRA, OAS2, OAS3, PTPN6, LYN, VAMP8, IRF6, ITGB2, CD47, which were also upregulated in ovarian cancer. Subsequently, the Kaplan-Meier plotter was applied to explore the correlation between gene expression and prognostic value of ovarian cancer patients, overexpression of four genes was connected with poor prognosis of ovarian cancer patients, including OAS2, OAS3, ITGB2, CD47. After that we found the OAS2, OAS3, CD47 are moderate related to immune infiltration in ovarian cancer and have the highest degree of correlation with macrophages infiltration, among which ITGB2 is strongly correlated with M2 macrophages infiltration level, and may have strong correlations in the differentiation of macrophages, thus promoting ovarian carcinogenesis and leading to an unfavorable prognosis.

ITGB2 is a receptor on the surface of cell membrane, which involved in the interaction between cells and extracellular matrix, and has the ability of bi-directional signal transmission,working as focal adhesions or hemidesmosomes in the migration and activation of immune cells and the maturation of DC cells, affecting the immune response of the body. ${ }^{12,13}$ Studies have revealed that ITGB2 is highly expressed in tumor tissues such as osteosarcoma, ${ }^{14}$ promoting tumor metastasis via ITGB2/FAK ${ }^{15}$.In addition, studies have found that Tregs target adhere to DC cells through ITGB2, resulting in impaired antigen presentation ability, directly inhibits T-cell activation. ${ }^{16}$ Our findings revealed that ITGB2 may be involved in the immune response in ovarian carcinogenesis and progression, resulting in a poor prognosis in ovarian patients.

In conclusion, OAS2, OAS3, ITGB2, CD47 were overexpressed in ovarian cancer as to normal ovarian tissues, and overexpression of them was positively correlated with TAMs immune infiltration as well as unfavorable clinical outcome in ovarian cancer patients, among which ITGB2 correlated with TAMs infiltration level most. However, the role of these immune related genes in ovarian cancer remains unclear, relevant research is needed to validate our findings. Although current immunosuppressive therapy is not effective enough in the treatment of ovarian cancer patients as expected, these genes may function as a biomarker for efficacy prediction, monitoring immune- related adverse reactions even to screen appropriate patients fit immunotherapy.

\section{Declarations}

\section{Availability of data and material}


All data generated or analysed during this study are included in this published article.

Acknowledgements

No applicable.

Funding

None.

Author information

1 Department of Gynecology and Obstetrics, The Fifth Affiliated Hospital of Sun Yat-Sen University, Zhuhai 519000,Guangdong, China

2 Department of Gynecologic Oncology, State Key Laboratory of Oncology in South China, Collaborative Innovation Center for Cancer Medicine, Sun Yat-Sen University Cancer Center, Guangzhou 510060, Guangdong, China

Chanyuan Li, jihong Liu

2 Department of Gynecologic Oncology, State Key Laboratory of Oncology in South China, Collaborative Innovation Center for Cancer Medicine, Sun Yat-Sen University Cancer Center, Guangzhou 510060, Guangdong, China

Ting Wan, Ting Deng囚Junya Cao®He Huang

\section{Contributions}

Conceptualization, $\mathrm{HH}$ and $\mathrm{JL}$; investigation, $\mathrm{CL}$ and TW; data curation, TD and JC; resources, CL; supervision, TW; writing-original draft, CL and TW; writing-review and editing, JL. All authors read and approved the final manuscript.

Corresponding author

Correspondence to Jihong Liu, He Huang; email: liujih@mail.sysu.edu.cn, huangh@sysucc.org.cn

Ethics declarations

Ethics approval and consent to participate

Not applicable.

Consent for publication

Not applicable. 


\section{Competing interests}

The authors declare no conflicts of interest.

\section{References}

1. Orr B, Edwards RP. Diagnosis and Treatment of Ovarian Cancer. Hematol Oncol Clin North Am. 2018;32(6):943-964.

2. Galon J, Bruni D. Approaches to treat immune hot, altered and cold tumours with combination immunotherapies. Nat Rev Drug Discov. 2019;18(3):197-218.

3. Yigit R, Massuger LF, Figdor CG, Torensma R. Ovarian cancer creates a suppressive microenvironment to escape immune elimination. Gynecol Oncol. 2010;117(2):366-372.

4. Ghisoni E, Imbimbo M, Zimmermann S, Valabrega G. Ovarian Cancer Immunotherapy: Turning up the Heat. Int J Mol Sci. 2019;20(12).

5. Binnewies M, Roberts EW, Kersten $\mathrm{K}$, et al. Understanding the tumor immune microenvironment (TIME) for effective therapy. Nat Med. 2018;24(5):541-550.

6. Baci D, Bosi A, Gallazzi M, et al. The Ovarian Cancer Tumor Immune Microenvironment (TIME) as Target for Therapy: A Focus on Innate Immunity Cells as Therapeutic Effectors. International Journal of Molecular Sciences. 2020;21(9).

7. Hanahan D, Weinberg RA. Hallmarks of cancer: the next generation. Cell. 2011;144(5):646-674.

8. Yang $Y$, Yang Y, Yang J, Zhao X, Wei X. Tumor Microenvironment in Ovarian Cancer: Function and Therapeutic Strategy. Front Cell Dev Biol. 2020;8:758.

9. $<$ The Yin-Yang of tumor-associated macrophages in neoplastic progression and immune surveillance.pdf $>$.

10. Zanganeh S, Spitler R, Hutter G, Ho JQ, Pauliah M, Mahmoudi M. Tumor-associated macrophages, nanomedicine and imaging: the axis of success in the future of cancer immunotherapy. Immunotherapy. 2017;9(10):819-835.

11. Colvin EK. Tumor-associated macrophages contribute to tumor progression in ovarian cancer. Front Oncol. 2014;4:137.

12. Schittenhelm L, Hilkens CM, Morrison VL. $\beta 2$ Integrins As Regulators of Dendritic Cell, Monocyte, and Macrophage Function. Frontiers in Immunology. 2017;8.

13. Brücher BLDM, Jamall IS. Cell-Cell Communication in the Tumor Microenvironment, Carcinogenesis, and Anticancer Treatment. Cellular Physiology and Biochemistry. 2014;34(2):213-243.

14. Benedicto A, Marquez J, Herrero A, Olaso E, Kolaczkowska E, Arteta B. Decreased expression of the beta2 integrin on tumor cells is associated with a reduction in liver metastasis of colorectal cancer in mice. BMC Cancer. 2017;17(1):827.

15. Harburger DS, Calderwood DA. Integrin signalling at a glance. Journal of Cell Science. 2009;122(9):1472-1472. 
16. Varga G, Balkow S, Wild MK, et al. Active MAC-1 (CD11b/CD18) on DCs inhibits full T-cell activation. Blood. 2006;109(2):661-669.

\section{Tables}

Table1 Significantly enriched GO terms and KEGG pathways of DEGs, ITGB2-related pathways can be found in supplementary figure1.

\begin{tabular}{|lllll|}
\hline Category & Term & Description & count & P value \\
\hline CC term & GO:0070062 & extracellular exosome & 84 & $1.72 \mathrm{E}-06$ \\
\hline CC term & GO:0016324 & apical plasma membrane & 18 & $6.18 \mathrm{E}-04$ \\
\hline CC term & GO:0005923 & bicellular tight junction & 10 & 0.00679287 \\
\hline CC term & GO:0005911 & cell-cell junction & 12 & 0.00679287 \\
\hline CC term & GO:0016323 & basolateral plasma membrane & 12 & 0.00784018 \\
\hline CC term & GO:0016328 & lateral plasma membrane & 7 & 0.00784018 \\
\hline CC term & GO:0005886 & plasma membrane & 93 & 0.00784018 \\
\hline BP term & GO:0030198 & extracellular matrix organization & 17 & $2.65 \mathrm{E}-04$ \\
\hline KEGG Pathway & hsa05150 & Staphylococcus aureus infection & 10 & 0.000092506 \\
\hline KEGG Pathway & hsa04514 & Cell adhesion molecules (CAMs) & 14 & 0.000168989 \\
\hline KEGG Pathway & hsa04670 & Leukocyte transendothelial migration & 11 & 0.002908282 \\
\hline
\end{tabular}

Table2 Top ten hub genes with highest degree of connectivity 


\begin{tabular}{|llc|}
\hline Gene name & Gene description & Score \\
\hline PTAFR & platelet-activating factor receptor & 11 \\
\hline HLA-DRA & DRa major histocompatibility complex, class II, DR alpha & 11 \\
\hline OAS2 & 2'-5'-oligoadenylate synthetase 2, 69/71kDa & 9 \\
\hline OAS3 & 2'-5'-oligoadenylate synthetase 3 & 9 \\
\hline PTPN6 & protein tyrosine phosphatase, non-receptor type 6 & 9 \\
\hline ITGB2 & v-yes-1 Yamaguchi sarcoma viral related oncogene homolog & 9 \\
\hline IRF6 & integrin, beta 2 (complement component 3 receptor 3 and 4 subunit) & 8 \\
\hline
\end{tabular}

Table3 Relationships between ITGB2,OAS2/3,CD47 expression and various markers of immune cells. $\left({ }^{\star} P<0.01,{ }^{* *} P<0.001,{ }^{* \star *} P<0.0001\right)$ 


\begin{tabular}{|c|c|c|c|c|c|c|c|c|c|}
\hline \multirow[t]{2}{*}{ Description } & \multirow{2}{*}{$\begin{array}{l}\text { Gene } \\
\text { markers }\end{array}$} & \multicolumn{2}{|l|}{ ITGB2 } & \multicolumn{2}{|l|}{ OAS2 } & \multicolumn{2}{|l|}{ OAS3 } & \multicolumn{2}{|l|}{ CD47 } \\
\hline & & $\mathrm{R}$ & $P$ & $\mathrm{R}$ & $P$ & $\mathrm{R}$ & $P$ & $\mathrm{R}$ & $P$ \\
\hline \multirow[t]{4}{*}{ TAM } & CCL2 & 0.4 & $\star \star \star *$ & 0.1 & 0.037 & 0.11 & 0.02 & 0.19 & $\star \star \star \star$ \\
\hline & CCL5 & 0.51 & $\star \star \star *$ & 0.19 & $\star \star \star *$ & 0.17 & $\star \star$ & 0.13 & * \\
\hline & CD68 & 0.84 & $\star \star \star *$ & 0.3 & $\star \star \star *$ & 0.3 & $\star \star \star *$ & 0.11 & 0.023 \\
\hline & IL10 & 0.5 & 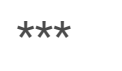 & 0.19 & $\star \star \star *$ & 0.16 & $\star *$ & 0.089 & 0.067 \\
\hline \multirow{5}{*}{$\begin{array}{l}\text { M1 } \\
\text { Macrophage }\end{array}$} & CXCL10 & 0.26 & 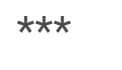 & 0.53 & 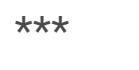 & 0.51 & $\star \star \star \star$ & 0.14 & * \\
\hline & TNF & 0.27 & $\star \star \star *$ & 0.0062 & 0.2 & 0.084 & 0.084 & 0.21 & $\star \star \star *$ \\
\hline & $\begin{array}{l}\text { INOS } \\
\text { QNOS2】 }\end{array}$ & -0.0015 & 0.98 & -0.0026 & 0.96 & -0.012 & 0.81 & -0.13 & * \\
\hline & IRF5 & 0.44 & $\star \star \star *$ & 0.29 & $\star \star \star *$ & 0.35 & $\star \star \star *$ & 0.079 & 0.1 \\
\hline & CD80 & 0.53 & $\star \star \star *$ & 0.52 & $\star \star \star$ & 0.52 & $\star \star \star$ & 0.13 & * \\
\hline \multirow{4}{*}{$\begin{array}{l}\text { M2 } \\
\text { Macrophage }\end{array}$} & CD163 & 0.68 & 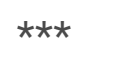 & 0.23 & $\star \star \star *$ & 0.21 & $\star * \star$ & 0.2 & $\star \star \star *$ \\
\hline & VSIG4 & 0.75 & $\star \star \star *$ & 0.24 & $\star \star \star *$ & 0.22 & $\star \star *$ & 0.13 & * \\
\hline & MS4A4A & 0.72 & 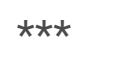 & 0.27 & $\star \star \star *$ & 0.23 & $\star \star \star *$ & 0.078 & 0.11 \\
\hline & $\begin{array}{l}\text { CD206 } \\
\text { बMRC1】 }\end{array}$ & 0.56 & 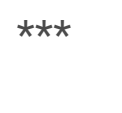 & 0.074 & 0.13 & 0.089 & 0.065 & -0.044 & 0.36 \\
\hline CD4+ & CD127 & 0.64 & $\star \star \star *$ & 0.12 & 0.015 & 0.15 & * & 0.14 & * \\
\hline \multirow[t]{2}{*}{ Tcell } & GITR & 0.16 & * & 0.074 & 0.14 & 0.13 & * & 0.11 & 0.024 \\
\hline & Foxp3 & 0.52 & $\star \star \star *$ & 0.36 & $\star \star \star *$ & 0.39 & $\star \star \star *$ & 0.17 & $\star \star$ \\
\hline CD8+ & CD8A & 0.47 & $\star \star \star *$ & 0.13 & * & 0.13 & * & 0.042 & 0.39 \\
\hline \multirow[t]{2}{*}{ Tcell } & CD8B & -0.022 & 0.65 & 0.055 & 0.26 & 0.065 & 0.18 & -0.063 & 0.19 \\
\hline & CD28 & 0.53 & $\star \star \star *$ & 0.16 & * & 0.18 & ** & -0.012 & 0.81 \\
\hline \multirow{3}{*}{ B cell } & CD19 & -0.044 & 0.37 & 0.11 & 0.028 & 0.12 & 0.016 & -0.048 & 0.33 \\
\hline & CD5 & 0.47 & $\star \star * *$ & 0.16 & * & 0.19 & $\star \star * *$ & 0.018 & 0.71 \\
\hline & CD79A & 0.23 & 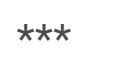 & 0.064 & 0.19 & 0.04 & 0.41 & -0.087 & 0.072 \\
\hline
\end{tabular}



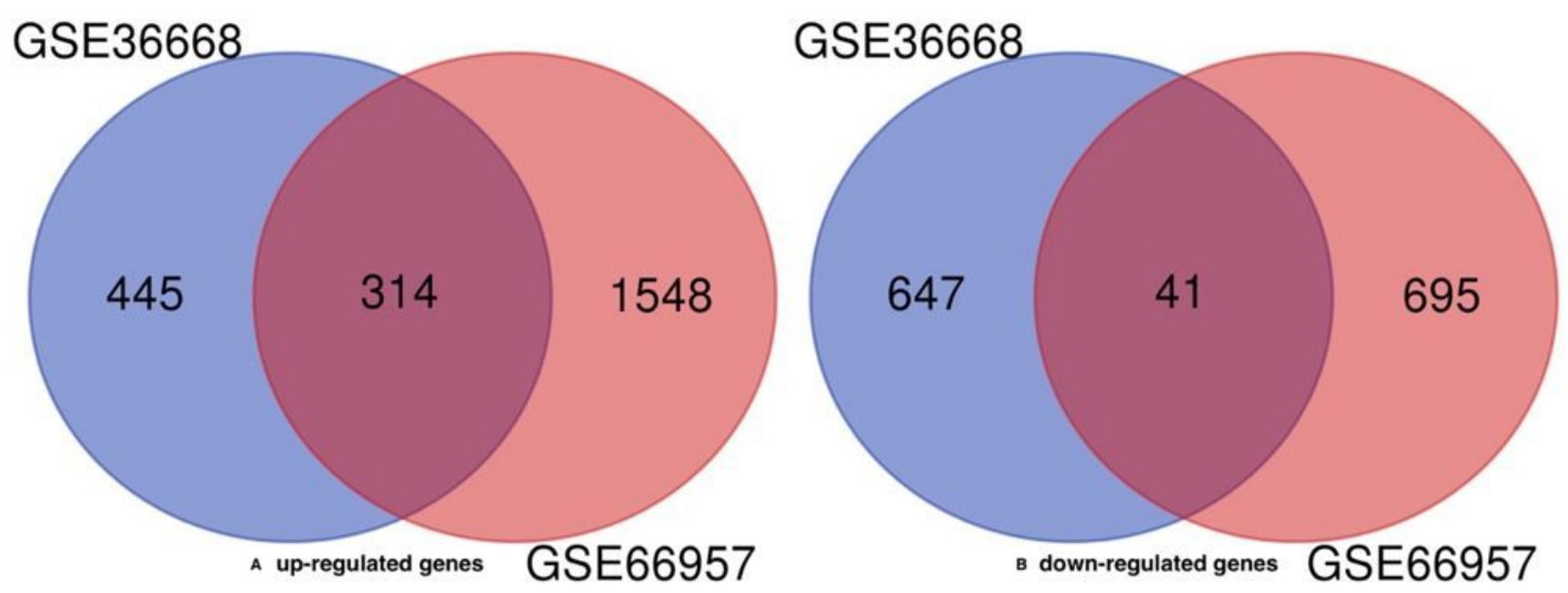

\section{Figure 1}

DEGs of two expression profiles (A up-regulated genes,B down-regulated genes)

A

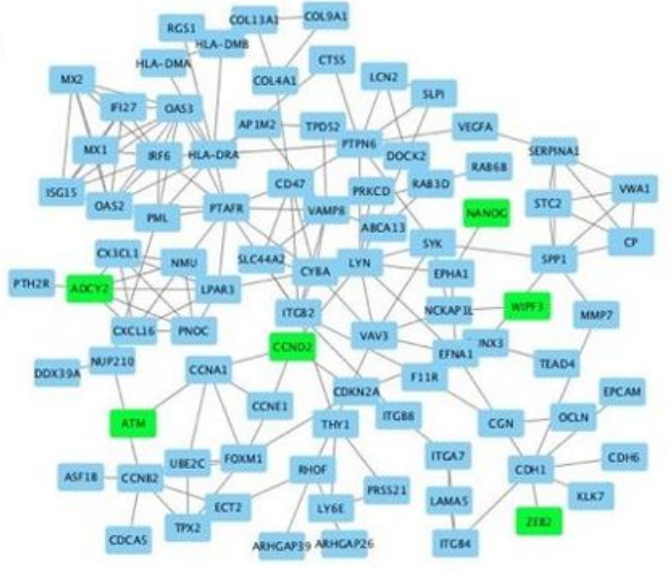

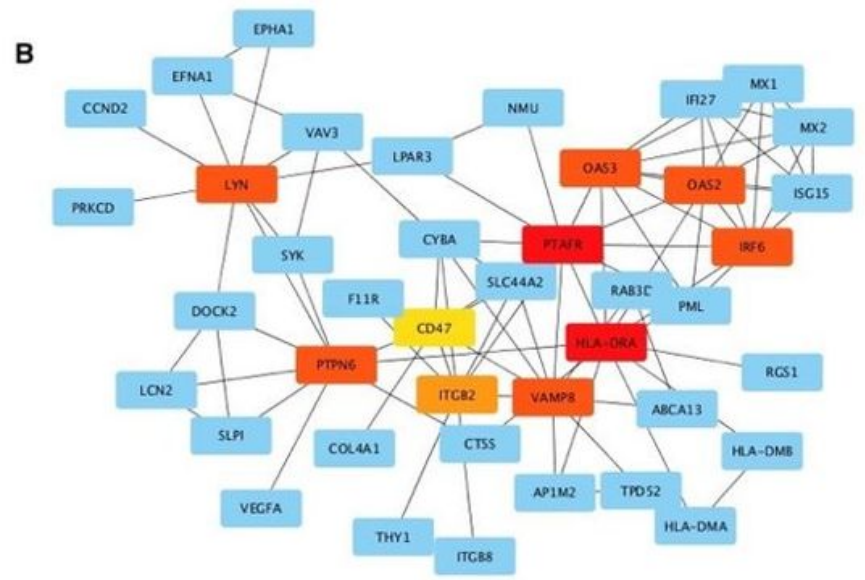

Figure 2

PPI network of connected nodes(A high degree connected 89 nodes, among which blue nodes stand for up-regulated genes, green nodes represent up-regulated genes.B 10 highest degree nodes). 

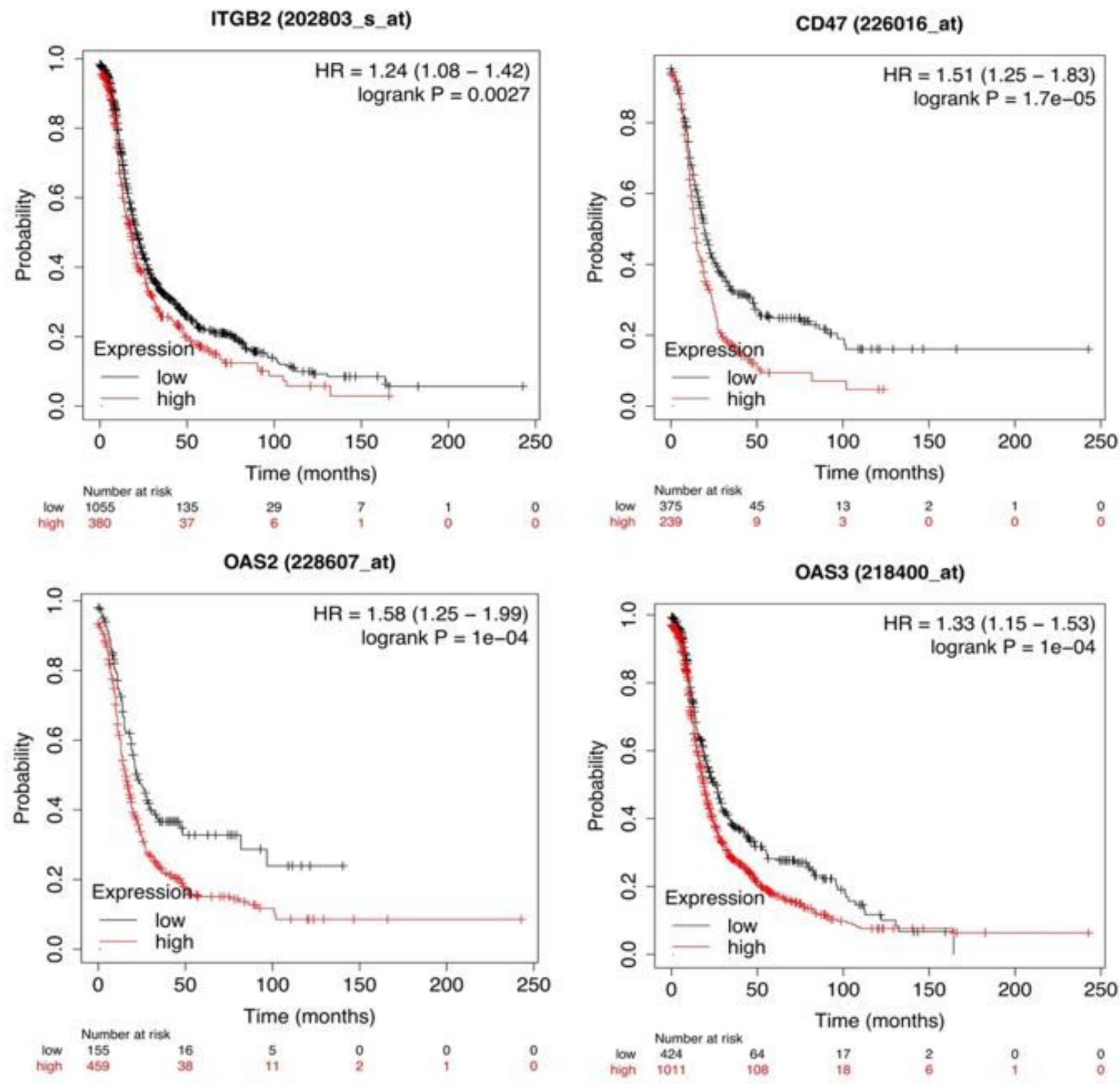

\section{Figure 3}

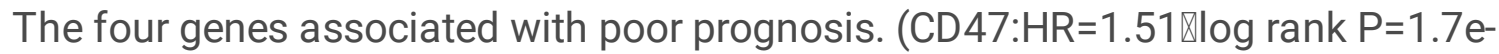
05,ITGB2:OAS2:HR=1.24,log rank $\mathrm{P}=0.0027,0 \mathrm{AS} 3: \mathrm{HR}=1.33$,log rank $\mathrm{P}=1 \mathrm{e}-04, \mathrm{OAS} 2: \mathrm{HR}=1.58$, og rank $\mathrm{P}=1 \mathrm{e}-04)$ 


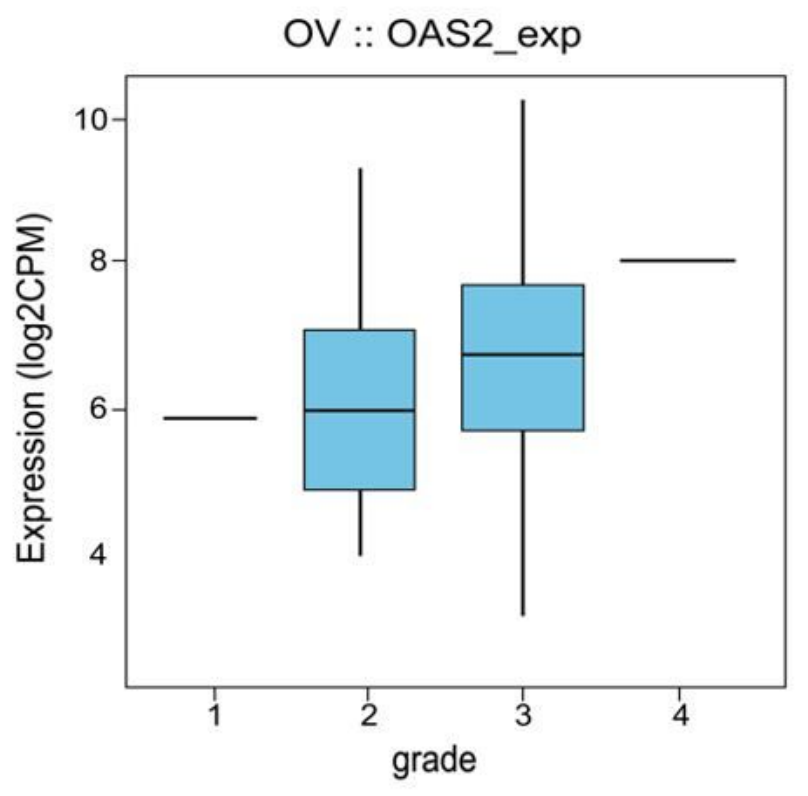

Spearman: $r h o=0.135, p=0.0201$

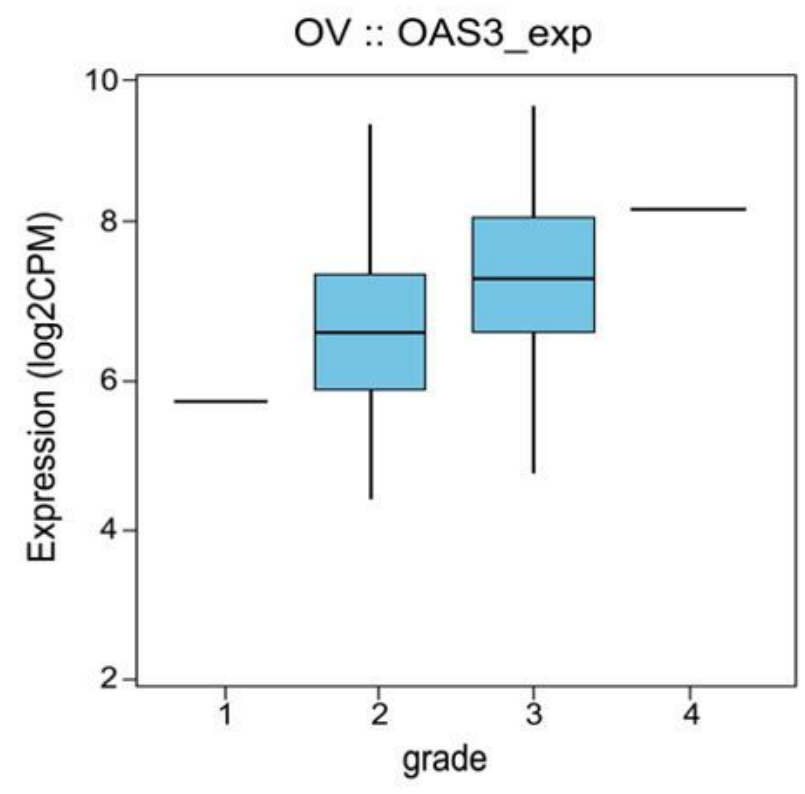

Spearman: $r h o=0.178, p=0.00211$

\section{Figure 4}

Expression of OAS2 and OAS3 are associated with higher grade in ovarian cancer. (OAS2:R=0135,P=0.0201;OAS3: $R=0178, P=0.00211$ )
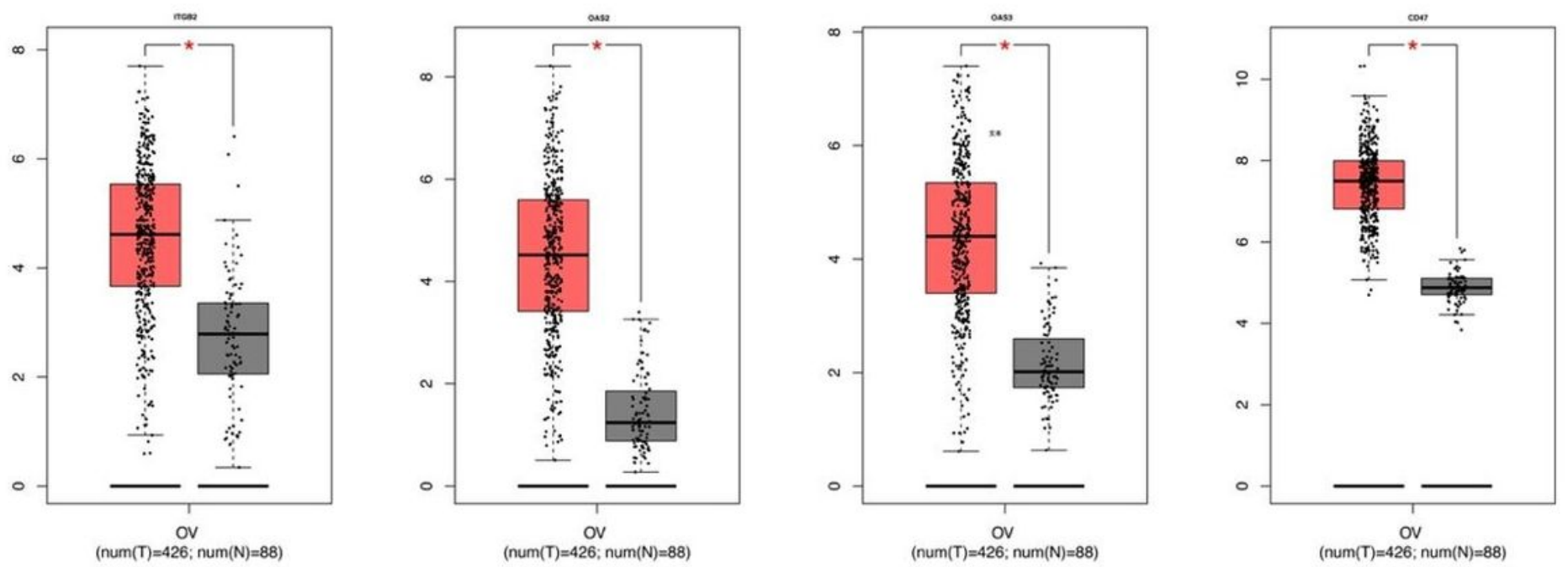

Figure 5

Expression of ITGB2,OAS2/3,CD47 in ovarian cancer samples compared to normal ovarian samples $\left({ }^{*} \mathrm{p}<0.05\right)$. 

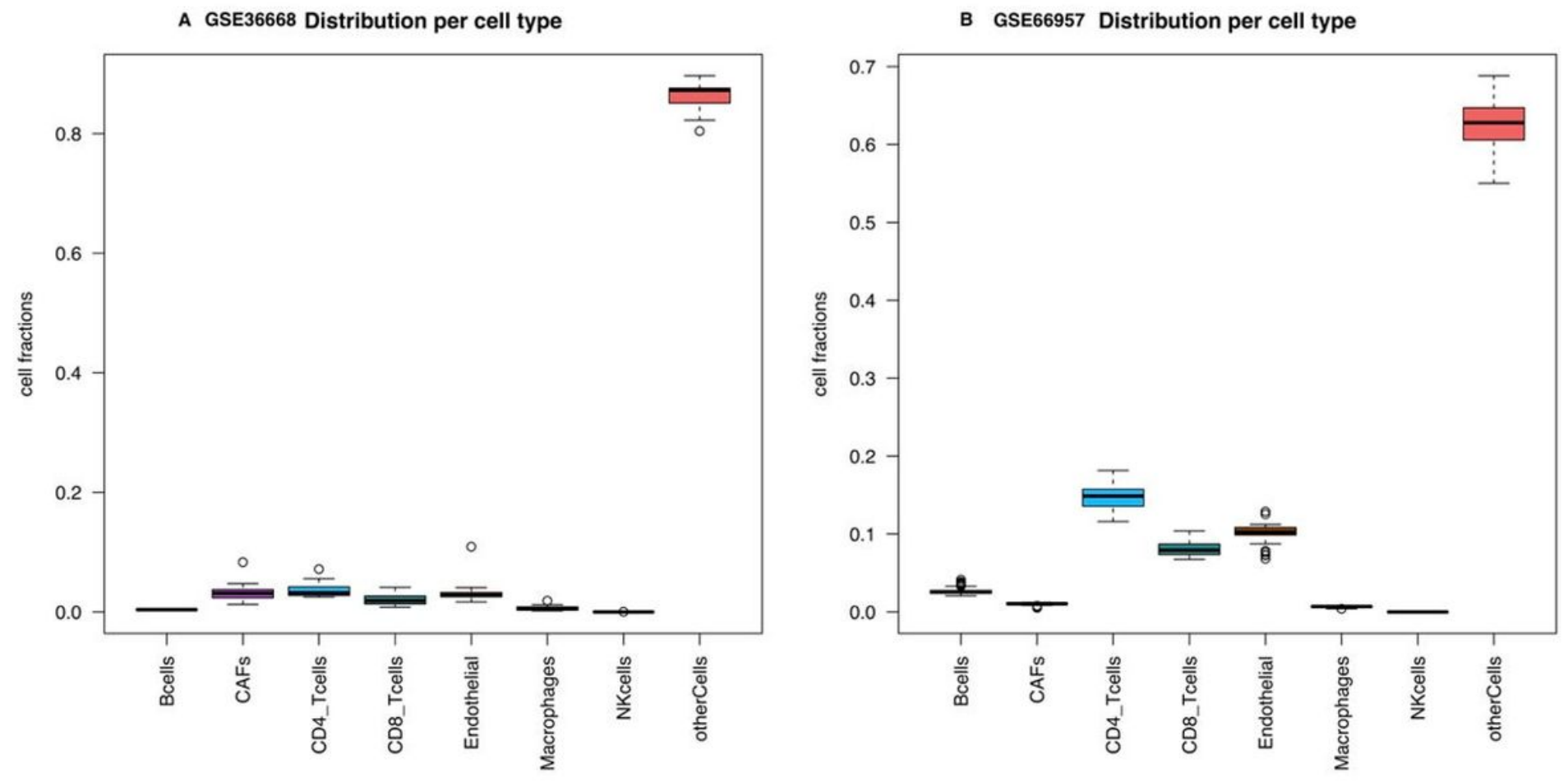

Figure 6

Tumor-infiltrating immune cell types and proportion in two profiles 

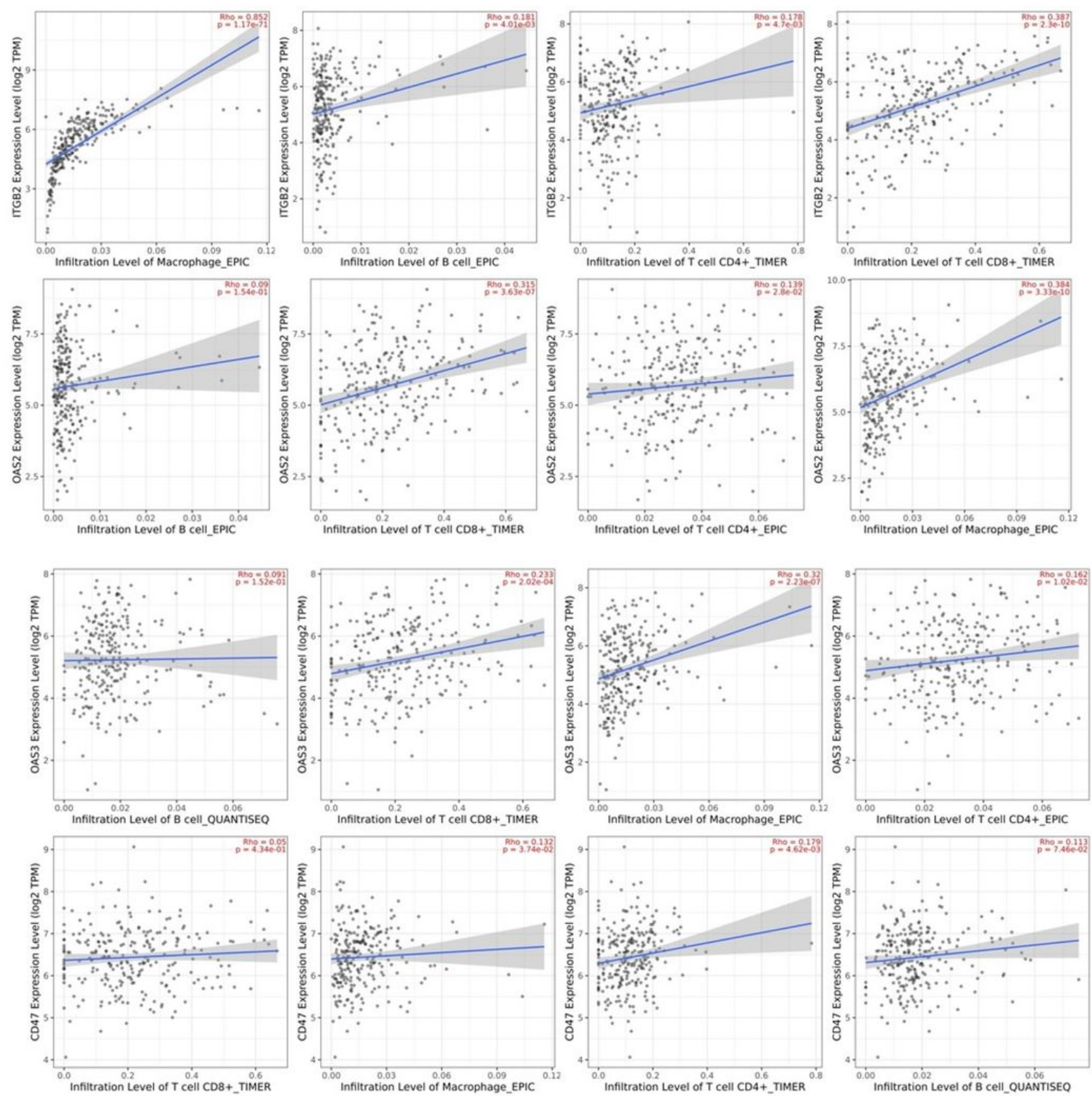

Figure 7

Correlation between ITGB2,OAS2/3,CD47 and major infiltrating immune cells(Macrophages, Bcell,CD4+ Tcell,CD8+ Tcell ). 

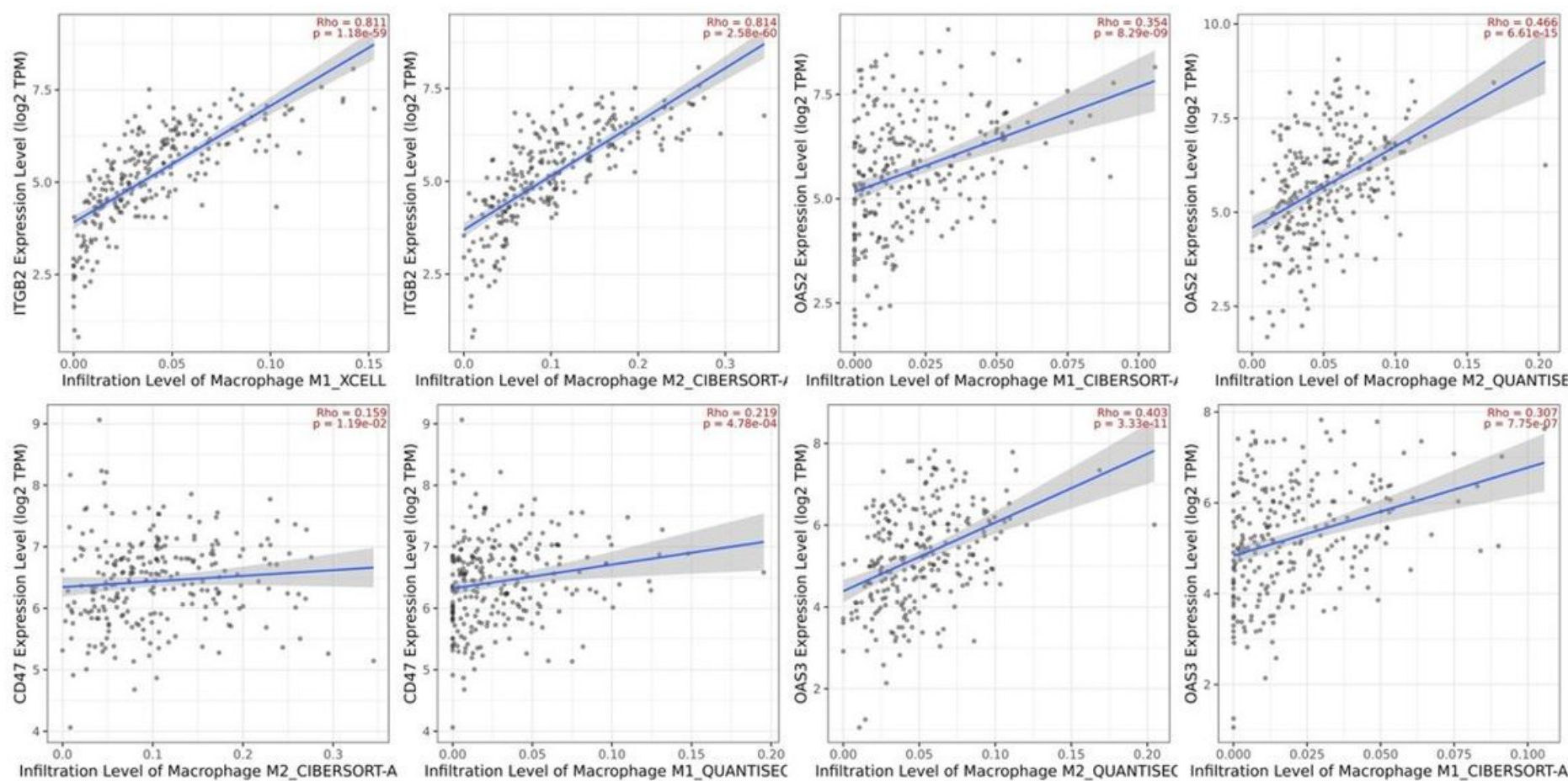

Figure 8

Correlation between ITGB2,OAS2/3,CD47 and M1 and M2 cells. 


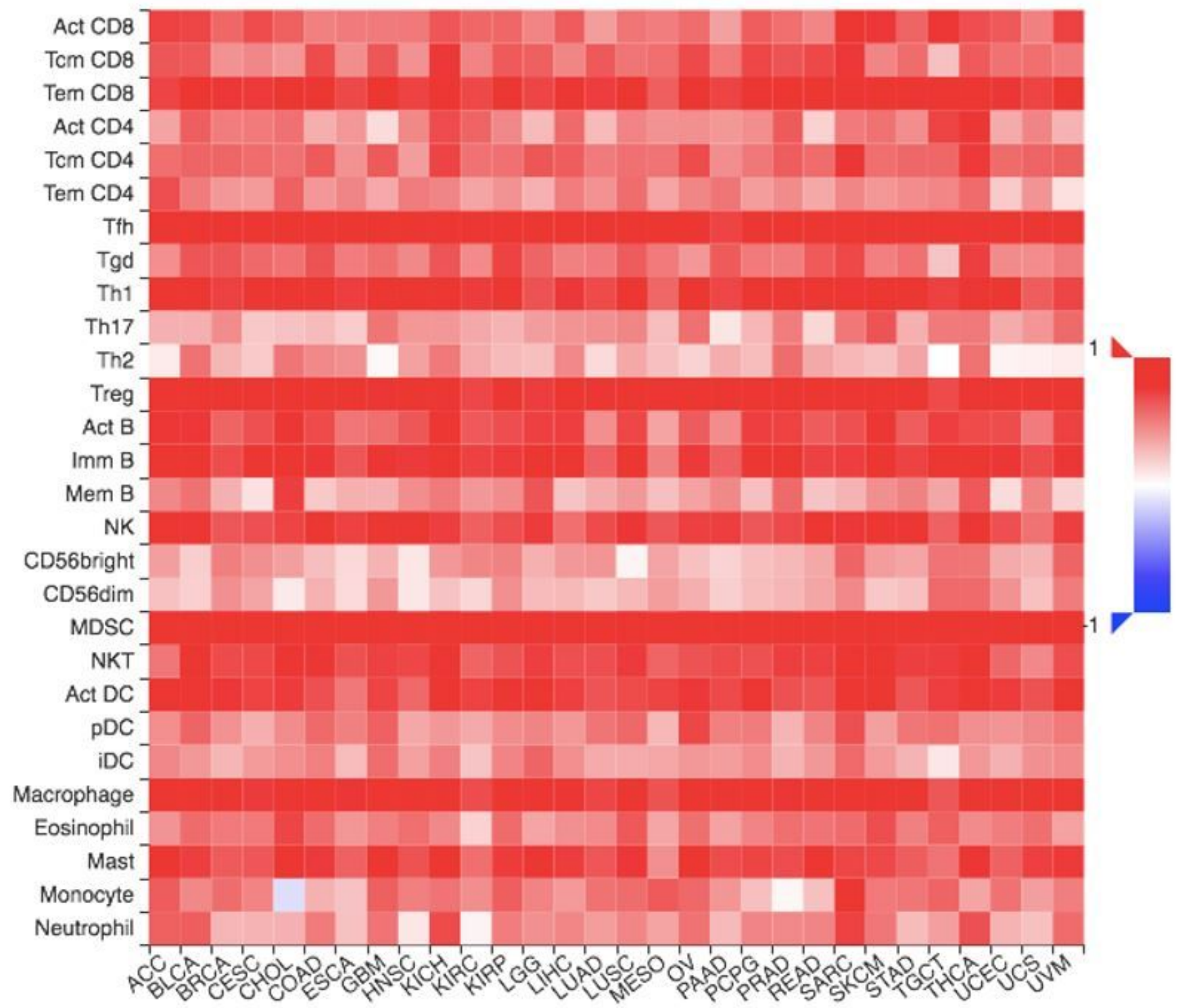

Figure 9

Correlation between ITGB2 and immune cells in human tumor.

\section{Supplementary Files}

This is a list of supplementary files associated with this preprint. Click to download.

- FigS1.jpg 